\title{
Alkaline glucose oxidation on nanostructured gold electrodes
}

\author{
M. Pasta ${ }^{1 *}$, R. Ruffo ${ }^{2}$, E.Falletta ${ }^{1}$, C.M. Mari ${ }^{2}$, C.Della Pina ${ }^{1}$ \\ www.goldbulletin.org
}

\begin{abstract}
The electrocatalytic properties of nanostructured gold electrodes for glucose electro-oxidation in $\mathrm{KOH}$ were investigated by cyclic voltammetry and compared with a commercially available polycrystalline gold electrode. These electrodes were prepared by depositing gold nanoparticles from a sol onto different carbonaceous conductive supports: glassy carbon, carbon cloth and graphite paper. The gold sol was prepared reducing an aqueous solution of tetrachloroauric acid with sodium borohydride. In order to improve gold nanoparticle adhesion, the substrate surfaces were treated with warm concentrated nitric acid. Gold on treated carbon cloth turned out to be a very promising anode for glucose electro-oxidation. In order to better understand the glucose oxidation its $\mathrm{pH}$ dependence as well as sorbitol (the glucose reduction product) electroxidation were investigated.
\end{abstract}

\footnotetext{
Dipartimento di Chimica Inorganica, Metallorganica e Analitica "Lamberto Malatesta", Università degli Studi di Milano, Via Venezian 21, 20133 Milano

2 Dipartimento di Scienza dei Materiali, Università degli Studi di Milano Bicocca, Via Cozzi 53, 20135 Milano

*Corresponding author: mauro.pasta@unimi.it
}

Keywords: Gold nanoparticle, direct glucose oxidation, nanostructured electrode, cyclic voltammetry

\section{Introduction}

The electro-oxidation of glucose has attracted a lot of interest due to its potential application in several areas, such as the development of blood sugar sensors and biological fuel cells [1, 2]. There are three main ways to perform glucose electro-oxidation, depending on the presence of active oxidant agents or mediators: microbe-assisted oxidation, enzymeassisted oxidation and direct oxidation at the electronic/ionic conductor interface.

Although the direct oxidation has several advantages, such as easy electrode preparation, long device lifetime and high thermodynamic efficiency in fuel cell applications, it has not been well investigated, and only a few significant works address this subject $[3,4]$.

Glucose electro-oxidation was firstly studied about one hundred years ago in a sulphuric acid electrolyte using a lead electrode [5]. Subsequent efforts were devoted to the investigation of electrocatalytic surfaces and achieving an understanding of the oxidation mechanism [6-10]. From those preliminary investigations it was discovered that the reaction depends highly on electrode materials and the crystalline orientation of their surfaces. Glucose oxidation on a bare gold electrode was investigated in the past by the polarization method, cyclic voltammetry $[6,8-10]$ and more recently by large amplitude Fourier transformed alternating current voltammetry [11]. It has been demonstrated that, among different materials, glucose oxidation is most active in alkaline solutions [6]. Moreover, it has been demonstrated that gold activity is strongly influenced by the $\mathrm{pH}$ and composition of the electrolyte solution. In a weak alkaline solution $(7<\mathrm{pH} \leq 10.5)$ the capability of gold to oxidize glucose depends mainly on the presence of an appropriate buffer solution [8] while no results are reported by using 
diluted alkali. From the reported studies, it became evident that the role of the buffering compounds (either phosphate or carbonate) is not merely the $\mathrm{pH}$ stabilization because their interaction with the sugar unit plays an important role. At higher $\mathrm{pH}(\geq 11)$ due to the presence of $\mathrm{KOH}$ gold is quite active and the dependence of the oxidation reaction with the $\mathrm{pH}$ has been investigated [6]. The oxidation of glucose in strong alkaline media is particularly interesting in sugar-fed fuel cell [12]. On the other hand the chemical instability of glucose during oxidation at high $\mathrm{pH}$ values and its degradation kinetics were widely investigated in the past years [13] showing the formation of complex mixtures of high and low molecular weight carboxylates and aldehydes rather than simple gluconate.

The proposed mechanism of glucose electroxidation on a gold electrode, based on the previous studies of gold redox and electrocatalytic behavior in aqueous media $[14,15]$, consists of fast chemisorption of glucose on the electrode, followed by dehydrogenation and simultaneous decomposition of the organic molecule [6]. An interesting aspect of glucose voltammograms on gold is the oxidative peak during the cathodic scan, [8-10] which has been explained by taking into account the role of oxygen anions desorbed from the metal surface [8].

More recently, several works have been devoted to the optimization of electrode performance in glucose sensor or fuel cell applications through chemical and morphological modification of gold electrodes [13, 16-19]. In particular, the highest efficiency of glucose oxidation has been obtained using modified gold nanoparticles at moderate alkaline $\mathrm{pH}$ values [18].

The aim of this work is to investigate the electrocatalytic performances of gold nanoparticles on glucose oxidation in alkaline solutions and to compare the results with those of commercial polycrystalline gold, in order to show the potential of these nanostructured electrodes as anodic catalytic material in glucose alkaline fuel cells (AFC). For this purpose, gold nanoparticles have been deposited on glassy carbon (GC) and on high surface area materials such as carbon cloth and graphite paper.

\section{Experimental}

Colloidal gold was prepared by adding $0.3 \mathrm{~mL}$ of a fresh $0.1 \mathrm{M} \mathrm{NaBH}_{4}$ (Aldrich) solution to $50 \mathrm{~mL}$ of $1 \mathrm{mM}$ tetrachloroauric acid solution (prepared by aqua regia dissolution of $99.9999 \%$ gold sponge, Aldrich) and glucose (50:1 molar ratio vs. $\mathrm{Au}$ ). Water was purified with a Milli-Q system. The nanostructured electrode was then obtained by adsorbing gold particles onto the carbonaceous materials by immersing in the solution and stirring for 12 hours as previously reported $[20,21]$. In the case of the Carbon tip (AMEL) electrode $(0.070$ $\mathrm{cm}^{2}$ ) the surface of the support was mechanical polished, sonicated in ethanol and rinsed with 2-propanol before deposition. Polycrystalline tip Au electrode (AMEL), with a surface area of $0.0314 \mathrm{~cm}^{2}$ was used for comparison. The deposition was also performed on larger $(30 \times 30 \mathrm{~mm})$ substrates of glassy carbon, (ALS Co., Ltd. CODE: 012095), carbon cloth and graphite paper (Hydro2Power S.r.l) by using the same deposition method. Moreover, the surfaces of the large substrates were treated with a strong acidic solution to increase the active area prior to gold nanoparticle deposition.

SEM images of nanoparticles on Glassy carbon plate were obtained at different magnification using a TESCAN VEGA TS5136XM electron microscope.

Electrochemical characterization was carried out using a PARSTAT 2263 potentiostat-galvanostat. A double junction $\mathrm{Ag}|\mathrm{AgCl}| \mathrm{KCl}$ (3.5M) reference electrode (RE) was used in the measurement. The double junction was employed to prevent $\mathrm{OH}^{-}$diffusion and reaction at $\mathrm{Ag} / \mathrm{AgCl}$ interface; additionally, the RE potential was monitored after each measurement to ensure consistency. All the potential values are reported vs. SHE. Before each measurement, the electrode surfaces were activated and stabilized in $0.25 \mathrm{M} \mathrm{H}_{2} \mathrm{SO}_{4}$ by $\mathrm{CV}$ scans at $100 \mathrm{mV} / \mathrm{s}$ between -0.4 and $1.2 \mathrm{~V}$ until stable voltammograms were observed (at least 20 scans). Glucose oxidation was performed by CV in different potassium hydroxide solutions ( $\mathrm{pH}=12-14$ ) with $10 \mathrm{mM}$ glucose using a platinum gauze counter electrode in a two compartment (glass frit) cell at scan rate of $100 \mathrm{mV} / \mathrm{s}$ from -1.0 to $0.5 \mathrm{~V}$. The two compartment cell was used to prevent the diffusion of by-products generated at the counter electrode to the working electrode. Before each electrochemical measurement, all the solutions were degassed by $\mathrm{N}_{2}$ purging for at least $1 \mathrm{~h}$.

\section{Results and discussion}

Nanoparticle morphology. The deposition of gold nanoparticles from sol provides low metal loading on glassy carbon support; no difference between 


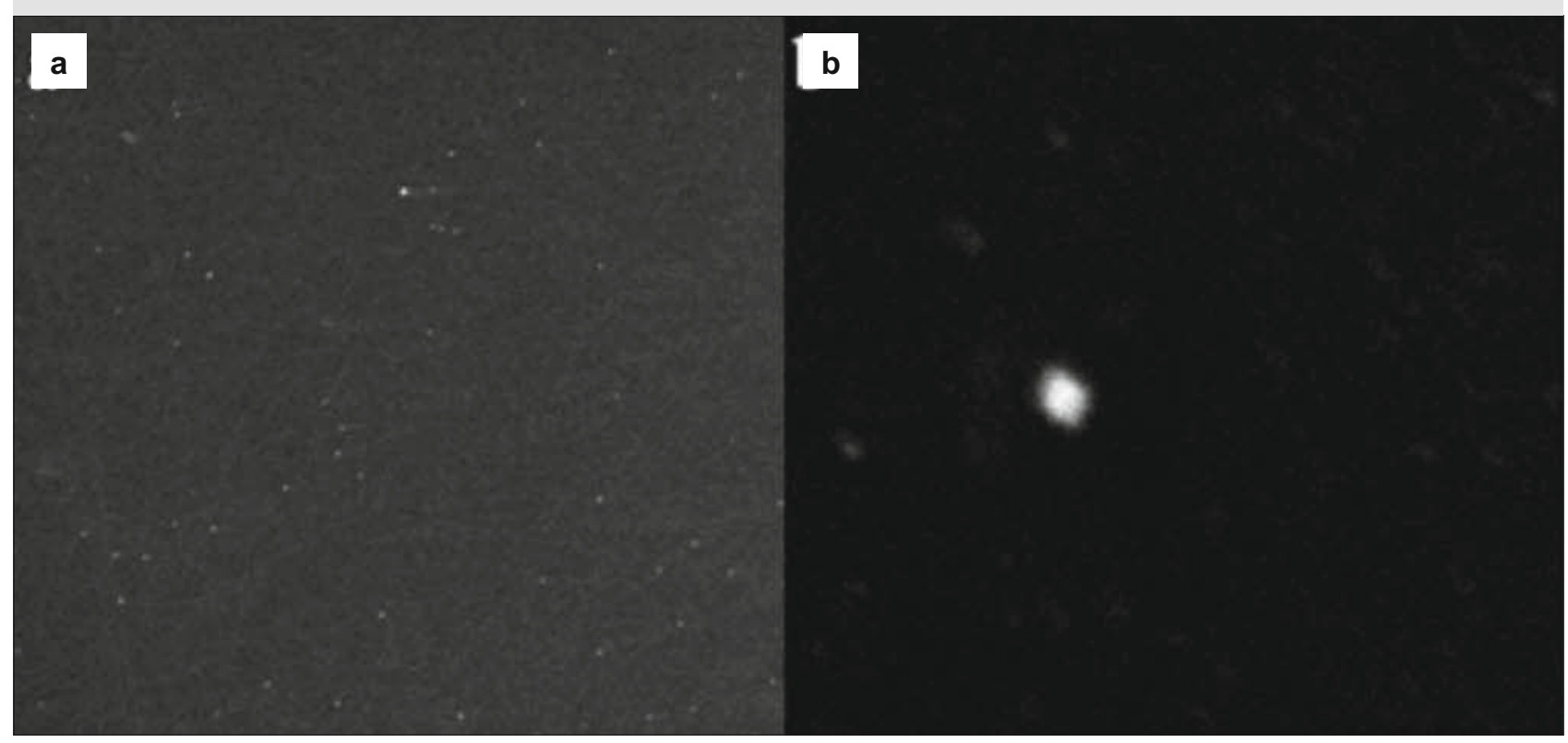

SEM images of gold nanostructured electrode: (a) 13.46kx, marker 10 $\mathrm{mm}$; (b) 144.59kx, marker $1 \mu \mathrm{m}$

loaded and pristine substrates is observable with the naked eye. The characterization of such a low gold surface area was very problematic: different electrochemical and physical methods (such as chronoamperometry, BET etc.) were unable to detect the small difference between impregnated and pristine surface areas.

Hence, SEM images were employed to evaluate particle distribution (Fig. 1a) and size (Fig. 1b) to roughly estimate the gold surface area. Assuming pseudo-spherical shape with average diameter of $100 \mathrm{~nm}$ (see Fig. 1b), and estimating a distribution of $12 \cdot 10^{6} \mathrm{Au}$ particle/ $\mathrm{cm}^{2}$, the gold surface would cover $0.1 \%$ of the glassy carbon plate. Thus, the nanosized gold active area is evaluated at $1 \cdot 10^{-4} \mathrm{~cm}^{2}$, which is approximately 300 times lower than the geometric area of polycrystalline electrode $\left(3.14 \cdot 10^{-2} \mathrm{~cm}^{2}\right)$.

\subsection{Electrochemistry of gold nanoparticles on glassy carbon tip}

The activation/stabilization CV scans of nanosized electrode material on a glassy carbon pin show the well-known behavior of gold when employed in a strong acidic solution [22] (Fig. 2 red curve). The oxidation process at high potential $(1.1 \mathrm{~V})$ is due to the formation of a superficial oxide layer on the gold surface; the layer is then reduced during the cathodic sweep at potentials around $0.65 \mathrm{~V}$. No significant differences were detected between

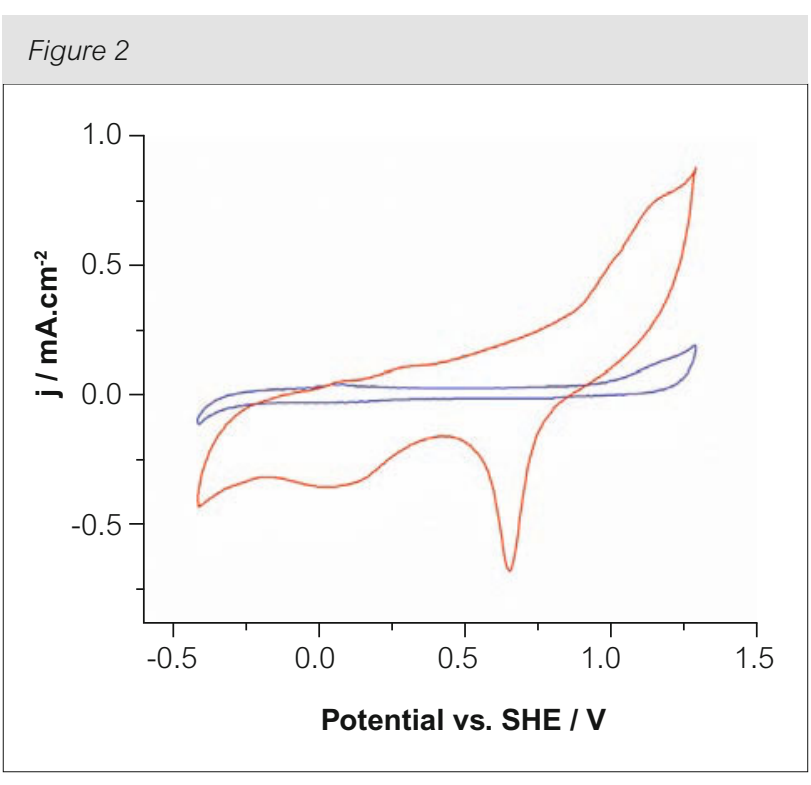

Cyclic voltammograms in $0.25 \mathrm{M} \mathrm{H}_{2} \mathrm{SO}_{4}$ of: (red curve) the gold nanoparticles on glassy carbon and (blue curve) pristine glassy carbon; scan rate $100 \mathrm{mV} / \mathrm{s}$, 20th cycle

two successive scans after 15 cycles. The unloaded glassy carbon electrode does not show electrochemical processes in the potential range investigated (Fig. 2 blue curve).

When the nanosized gold electrode is cycled in alkaline solution (Fig. 3 blue curve), two oxidation and one reduction processes are observed. The 
anodic peaks at 0.2 and $0.7 \mathrm{~V}$ are usually attributed to the $\mathrm{OH}^{-}$oxidative adsorption $[10,17]$ and the formation of oxide layer $\mathrm{Au}-\mathrm{O}$, respectively; $\mathrm{Au}-\mathrm{O}$ is then reduced at $-0.1 \mathrm{~V}$ during the cathodic scan [17].

The polycrystalline commercial electrode shows curves which are qualitatively similar in both acidic and alkaline solutions.

\subsection{Glucose oxidation by gold nanoparticles on glassy carbon tip}

The CV curve of the nanosized electrode in glucose alkaline solution shows three clear electrochemical processes during the anodic sweep and one during the cathodic return scan (Fig. 3, red curve). Peak "a" (-0.7 V) was attributed by many authors to dehydrogenation of anomeric carbon under adsorption control [23-25]. The dehydrogenation of the anomeric carbon peak was deeply investigated by Beden et al. on a platinum electrode [26, 27]. In particular, it was found that when the solution was prepared and kept at low temperature $\left(2^{\circ} \mathrm{C}\right)$ this peak became remarkably high; in fact at low temperature, in alkaline medium ( $\mathrm{NaOH} 0.1 \mathrm{M})$ the $\beta$-D-glucose quickly becomes the main species in solution (as determined by GC-MS). Voltammetric investigations proved that $\beta$-D-glucose is the most active anomer due to the planar approach of the molecule to the electrode surface. Furthermore it is important to note that this peak shifts about $600 \mathrm{mV}$ toward lower potential with respect to the polycrystalline platinum.

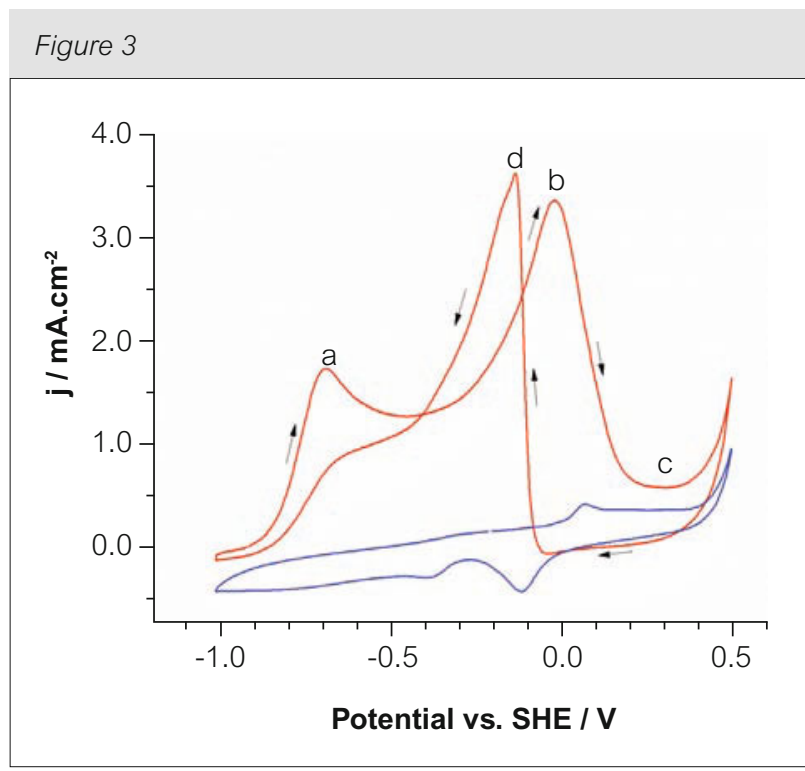

Cyclic voltammograms of the nanostructured gold electrode in: $0.1 \mathrm{M} \mathrm{KOH}$ (blue curve) and $0.1 \mathrm{M} \mathrm{KOH}$ plus $10 \mathrm{mM}$ glucose (red curve) solutions; scan rate $100 \mathrm{mV} / \mathrm{s}, 10$ th cycle
The higher oxidation potential on $\mathrm{Pt}$ is probably due to the hydrogen adsorption that competitively hinders glucose adsorption; this phenomenon is negligible on the gold surface.

A second electrochemical process can be observed at $0.0 \mathrm{~V}$ in Fig. 3 red curve, peak b. This peak has a large left shoulder due to several oxidative processes taking place in the potential range from -0.3 to $0.0 \mathrm{~V}$. Beden et al. described the processes on a Pt electrode at low temperature $\left(2^{\circ} \mathrm{C}\right)$ and found out that the oxidation of the previously adsorbed intermediate follows two possible routes: at $\mathrm{E}<0.1$ $\mathrm{V}$, it is oxidized as weakly adsorbed gluconate, either linked by two oxygen or by only one oxygen (at -0.2 $<E<0.1 \mathrm{~V}$ ), while the second route is observed at $0.1<\mathrm{E}<1.1 \mathrm{~V}$, when glucose is oxidized as weakly adsorbed $\delta$-gluconolactone. At $25^{\circ} \mathrm{C}$ we observed a convolution of all these peaks in peak b: it is evident however, that in both cases the oxidation product is always gluconate in these conditions.

Around 0.2 V (Fig. 3 red curve, zone c) gold surface oxidation occurs, as can be concluded comparison of the CV curves in the presence and absence of glucose. Xie et al [17] demonstrated the formation of a Au/oxygen species in the $0.2 \mathrm{~V}$ region by quartz crystal microbalance analysis coupled with electrochemical apparatus. Furthermore they pointed out that glucose can be oxidized at the metal gold surface but not at the Au oxide surface, so the current decreases at potentials higher than $0.3 \mathrm{~V}$.

The oxidative peak in the $\mathrm{CV}$ reductive scan (Fig. 3 red curve, peak d), previously observed in several reported studies, $[10,17,18]$ is obtained as soon as the oxide layer is reduced. The mechanism for the oxidation during the cathodic sweep was first proposed by Liu and Makovos: the oxide layer is reduced to generate free $\mathrm{O}^{2-}$ anions which react with glucose to give gluconic acid, and then the Au surface undergoes re-oxidation by $\mathrm{OH}^{-}$in solution [10]. No electrochemical processes were detected in the glucose solution using the unloaded glassy carbon electrode (Fig. 3, blue curve).

\subsection{Comparison between nanoparticles and commercial gold pin electrode}

When compared to the commercial polycrystalline electrode, nanostructured gold particles exhibit better performance in terms of current density output, and also a shift towards lower potentials of the oxidation processes (Fig. 4). It is important to emphasize that in both cases the current densities 
were referenced to the geometrical surface area. In the case of the polycristalline gold pin, the real surface area was measured by chronoamperometry [28] using different electroactive species $\left(\mathrm{Fe}(\mathrm{CN})_{6}{ }^{4-}, \mathrm{Fe}(\mathrm{CN})_{6}{ }^{3-}\right.$ and $\left.\mathrm{Ag}^{+}\right)$: no difference with respect to the geometrical one was discovered. Since the effective area of the gold nanoparticles is about one hundred times lower, as previously discussed, the actual current density of the nanoparticle electrode should be at least two orders of magnitude higher. Moreover it is interesting to observe the large increment in the peak (see Fig. 3) current density, attributed to the presence of more defective sites on the surface of gold nanoparticles. It is well known that surface reactions usually require lower activation energy in presence of defective sites (i.e. kink atom, adatom, edge adatom, etc.); as a consequence, the nanoparticles should have an intrinsic large number of defects which are responsible for increased glucose oxidative adsorption.

\section{$3.4 \mathrm{pH}$ effect on glucose oxidation}

In order to understand the role of $\mathrm{pH}$ on glucose electro-oxidation we first investigated the behavior of a commercial gold electrode. CV scans of $\mathrm{KOH}$ electrolyte solutions (Fig. 5) show that the oxidation of the gold surface depends slightly on the $\mathrm{pH}$ as well as its onset and the peak position lay around $-0.35 / 0.0$ and $0.05 / 0.10 \mathrm{~V}$, respectively. As the $\mathrm{pH}$ increases, the glucose oxidation peak $b$ moves towards lower potentials, meaning that the reaction becomes more favorable, while the adsorption peak

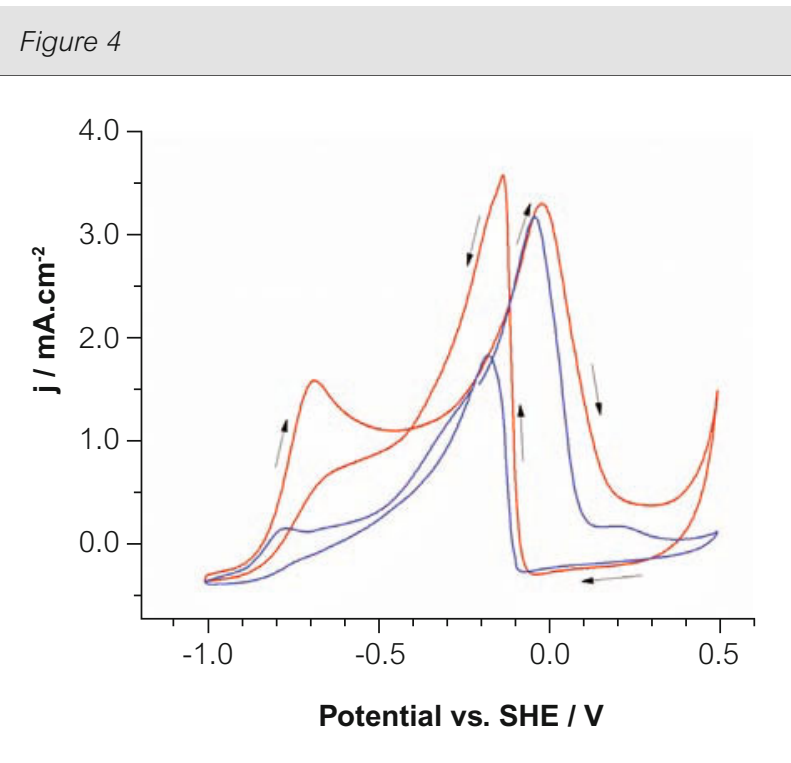

Cyclic voltammograms in $10 \mathrm{mM}$ glucose, $0.1 \mathrm{M} \mathrm{KOH}$ solution of: polycrystalline (blue curve) and nanostructured (red curve) gold electrode; scan rate $100 \mathrm{mV} / \mathrm{s}$, 10 th cycle

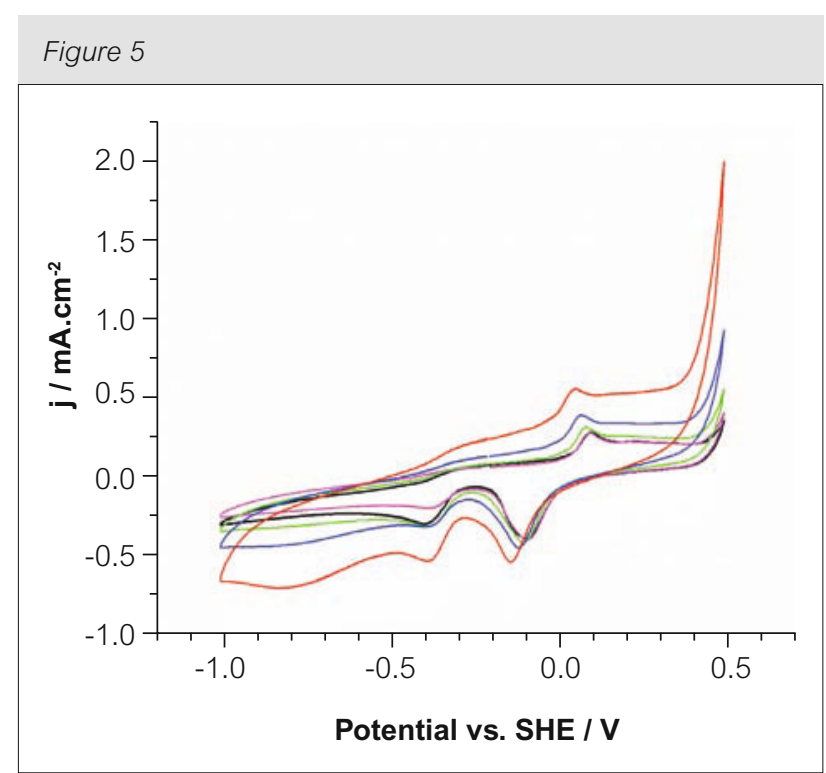

Cyclic voltammograms of the gold electrode at different $\mathrm{pHs:}$ $($ red curve $)=14.0,($ blue curve $)=13.5,($ green curve $)=13.0$, ( purple curve $)=12.5$, (black curve $)=12.0$ scan rate $100 \mathrm{mV} / \mathrm{s}$, 10th cycle

a shows little $\mathrm{pH}$ dependence (Fig 6). The fact that the gold surface electrochemical process (peak a) is only slightly influenced by $\mathrm{pH}$ suggests that the strong $\mathrm{pH}$ dependence of glucose oxidation potential (peak b) is relative to an interaction with $\mathrm{OH}^{-}$in solution. This interaction is likely a nucleophilic attack by $\mathrm{OH}^{-}$ on the glucose carbonyl group in linear form, with the formation of an intermediate which interacts strongly with the Au electrode surface. These observations might indicate an electro-oxidation reaction like that proposed by Rossi et. al, [20] where the chemical oxidation of glucose in the presence of molecular oxygen involves a two electron mechanism, with the formation of gluconate and hydrogen peroxide.

\subsection{Sorbitol electrochemistry}

Sorbitol is the reduction product of glucose in which the aldehydic group is substituted with the alcoholic function. Due to the lack of the carbonilic group, sorbitol cannot form the closed six atom ring configuration and it does not have any anomeric carbon. It seems, therefore, interesting to compare sorbitol and glucose oxidation on the same electrode used in 3.4. The comparison between the CV of the two molecules (Fig. 7) illustrates the absence of the low potential process in the case of sorbitol, thus confirming our previous conclusion regarding peak a in figure 3 . Sorbitol shows a large oxidation peak at $0.1 \mathrm{~V}$, the same potential value attributed to the glucose peak $b$ in figure 3 . The peak shape is very similar, however the current density of the process 


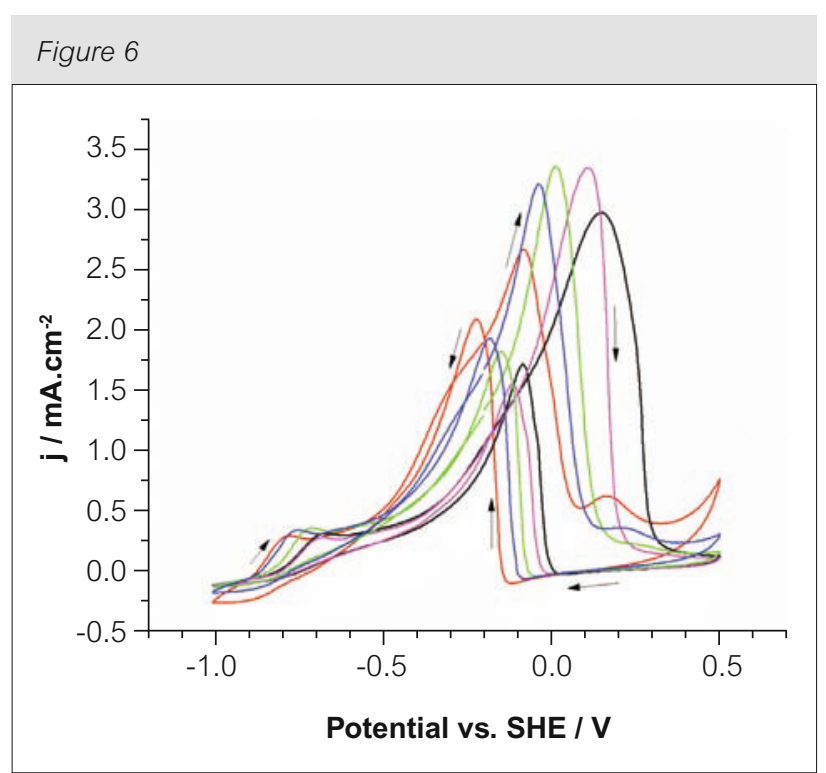

Cyclic voltammograms in $10 \mathrm{mM}$ glucose, $0.1 \mathrm{M} \mathrm{KOH}$ solution of the polycrystalline gold electrode at different $\mathrm{pHs}$ : (red curve) = 14.0, (blue curve $)=13.5$, (green curve $)=13.0$, (purple curve $)=$ 12.5, (black curve) $=12.0$; scan rate $100 \mathrm{mV} / \mathrm{s}, 10$ th cycle

is almost twofold; this could be due to the oxidation of the primary $-\mathrm{CH}_{2} \mathrm{OH}$ functional groups, since there are two in sorbitol, but just one in glucose. Due to sorbitol's remarkable chemical stability in strong alkaline solutions, it allows a better analysis of the uncontaminated oxidation products after electrolysis of sorbitol solution at $0.1 \mathrm{~V}$. Preliminary HPLC analysis show that electrochemical oxidation of sorbitol produces not only gluconic acid, the most abundant product, but also other chemical species.

\subsection{Glucose oxidation on different carbonaceous conductive supports: effect of a nitric acid treatment}

To test the glucose oxidation on large electrodes for practical applications, we have investigated the electrochemistry of different carbon based substrates impregnated with the gold nanoparticles. The low metal loading obtained with the deposition method is probably due to the very smooth and hydrophobic surface of the GC support, which reduces the nanoparticle adhesion and explains the presence of particle agglomerations.

In order to improve the gold surface area three different carbonaceous conductive supports (GC plate, carbon cloth and graphite paper) were studied and compared. All the supporting materials underwentatreatment with warm $\left(70^{\circ} \mathrm{C}\right)$ concentrated $(65 \%)$ nitric acid before the subsequent deposition of colloidal gold sol.
It is known that oxidation of carbon surfaces can offer, not only a more hydrophilic surface structure, but also a larger number of oxygen-containing functional groups as quantitatively measured by Boehm's method [29]. Among different oxidant agents (such as hydrogen peroxide and potassium permanganate), nitric acid is considered the most active reagent for generating a larger number of carboxyl and lactone groups [30].

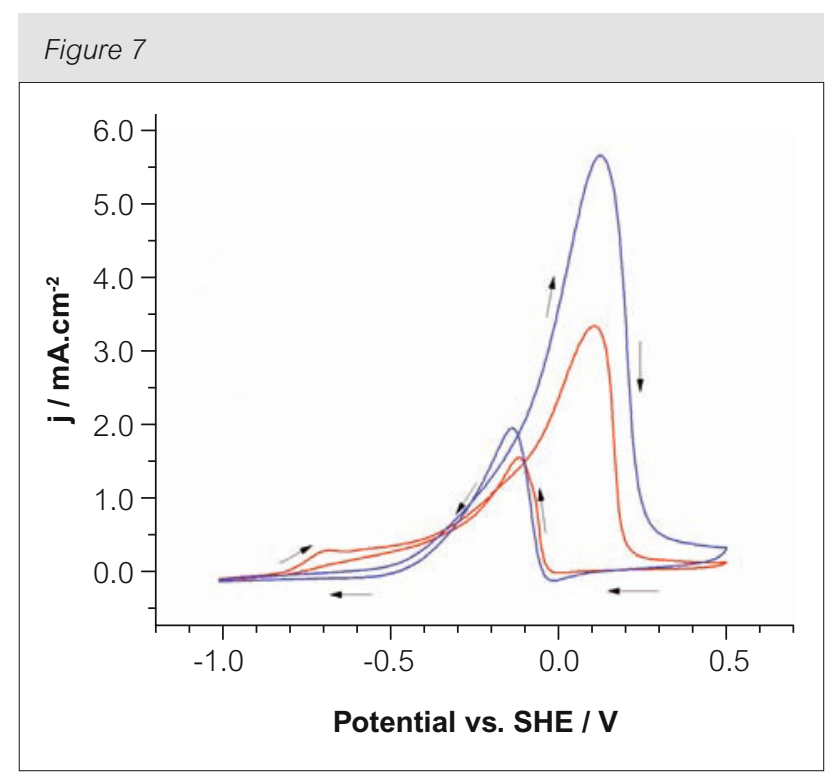

Cyclic voltammograms of polycrystalline gold electrode in 10 $\mathrm{mM}$ glucose, $0.1 \mathrm{M} \mathrm{KOH}$ solutions of: glucose (red curve) and sorbitol (blue curve); scan rate $100 \mathrm{mV} / \mathrm{s}, 10$ th cycle

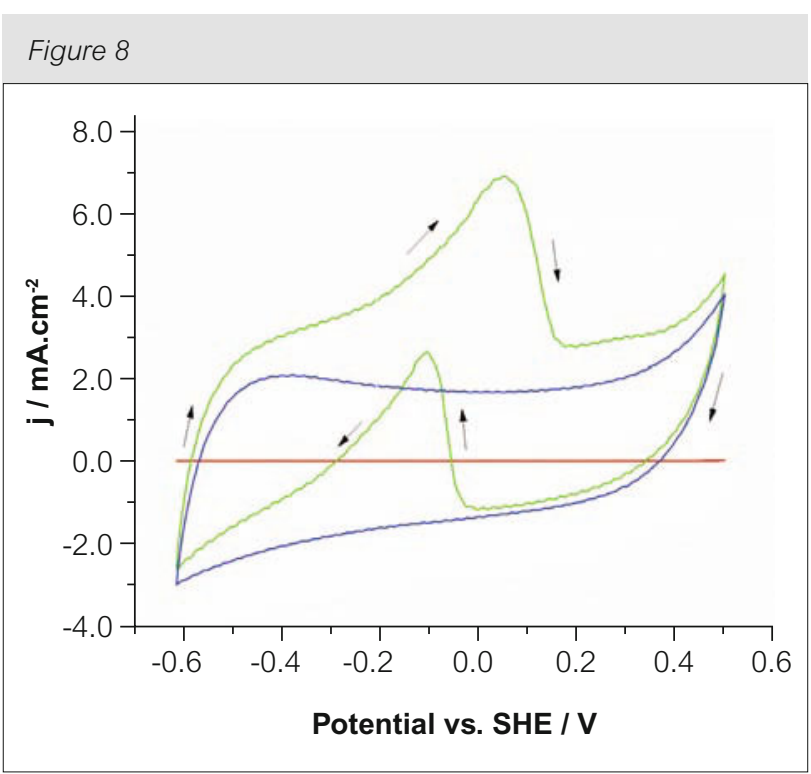

Cyclic voltammograms in $10 \mathrm{mM}$ glucose, $0.1 \mathrm{M} \mathrm{KOH}$ solution of: (red curve) pristine, (blue curve) nitric acid treated and (green curve) nitric acid treated with gold nanoparticles carbon cloth electrode; scan rate $100 \mathrm{mV} / \mathrm{s}, 10$ th cycle 
In figure 8 is reported the $\mathrm{CV}$ of pristine (red curve), treated (blue curve) and treated with gold nanoparticle (green curve) carbon cloth. Remarkable increase (one order of magnitude) of the current was observed for treated samples, probably due to the improved hydrophilicity of the support which permits a better contact between substrate and surface. The presence of gold particles further increases the current density.

Similar behavior was also observed for both glassy carbon and graphite paper.

In figure 9 the performances of the three carbonaceous conductive supports are compared after the nitric acid treatment and gold sol deposition; GC clearly shows the worst performance. The oxidative peak potential on both graphite paper and carbon cloth lies at around the same value $(0.0 \mathrm{~V})$, but the current density of the latter (about $7.0 \mathrm{~mA}$ ) $\mathrm{cm}^{2}$ ) is four times higher.

Gold nanoparticles on nitric acid treated carbon cloth appears to be a very promising anode material for glucose electro-oxidation.

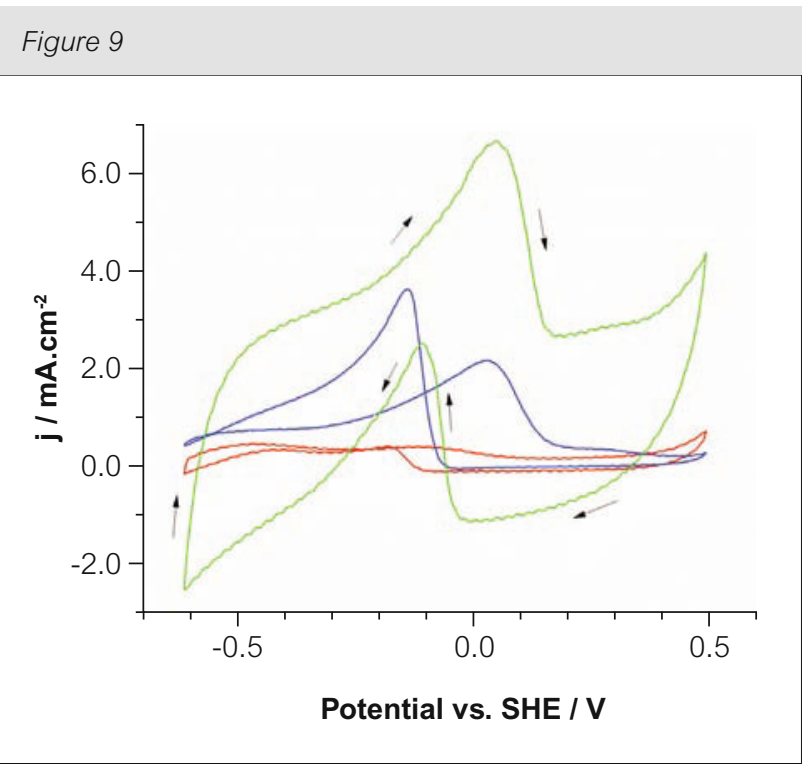

Cyclic voltammograms in $10 \mathrm{mM}$ glucose, $0.1 \mathrm{M} \mathrm{KOH}$ solution of nitric acid treated with gold particles: (red curve) glassy carbon, (blue curve) graphite paper, (green curve) carbon cloth electrode; scan rate $100 \mathrm{mV} / \mathrm{s}, 10$ th cycle

\section{Conclusion}

A nanostructured gold electrode was obtained on the surface of a glassy carbon tip by depositing the noble metal from a colloidal dispersion, prepared by aqueous cloroauric acid reduction. The gold particles (average size $100 \mathrm{~nm}$ and electrode surface area about $1 \cdot 10^{-4} \mathrm{~cm}^{2}$ ) exhibit better electrocatalytic properties with respect to commercially available polycrystalline electrode for glucose oxidation. The evaluation of the oxidation peak as a function of the $\mathrm{pH}$ illustrates the strong improvement of substrate reactivity obtained by increasing the $\mathrm{OH}^{-}$ concentration. Moreover, the oxidation of sorbitol, the reduced form of glucose, confirmed the assignment of the peak at $-0.75 \vee$ to the anomeric carbon oxidation.

A very strong increment of the electrode performance in glucose oxidation was obtained by improving the total amount of gold nanoparticles which was achieved using a carbon cloth-support. With this electrode the current densities are increased up to $7 \mathrm{~mA} / \mathrm{cm}^{2}$.

These results open new possibilities for gold in Glucose Alkaline Fuel Cell (GAFC) applications.

\section{About the authors}

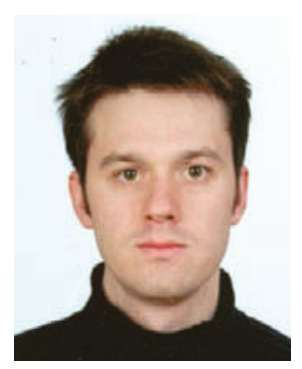

Mauro Pasta earned his master degree in Industrial Chemistry from the State University of Milan in 2007. He is working on his $\mathrm{PhD}$ thesis concerning gold catalysis applied to chemical and electrochemical oxidation of carbohydrates.

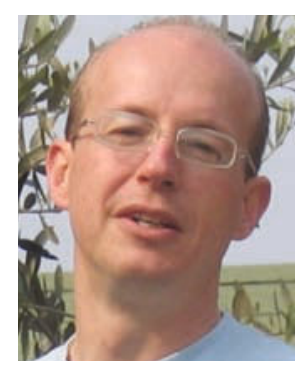

Riccardo Ruffo graduated in Chemistry in 1997 and obtained the Master in Material Science and Technology in 2000 at the State University of Milano. Awarded the Ph.D. in Material Science at the State University of Milano Bicocca in 2004. His research interests cover a wide range of applied electrochemistry. 


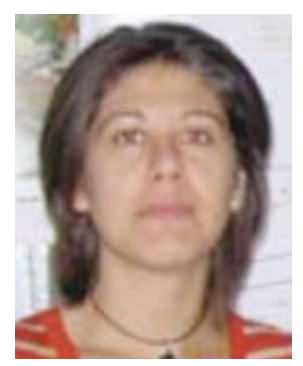

Ermelinda Falletta got her master degree in Organic Chemistry at the University of Palermo. In 2005 she joined Prof. Rossi's research group at Milano University involved in catalytic oxidation of organic compounds.

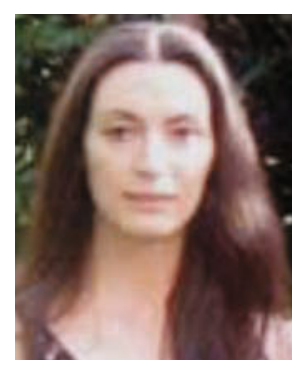

\section{Cristina Della Pina received the}

master degree at Milan University and awarded the Ph.D. degree in Industrial Chemistry in 2006. Her work focuses on the development of heterogeneous catalysts.

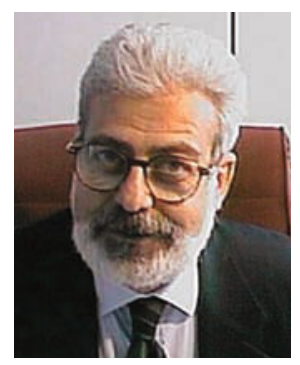

Claudio Maria Mari earned his degree in Industrial Chemistry from the State University of Milan. He is full professor of Physical Chemistry and teaches Electrochemistry and Corrosion in the Master of Materials Science and Physical Chemistry in the Bachelor of Chemical Science and Technology. Thermodynamics of non-stoichiometric oxides, Statistical Thermodynamics, Electrochemistry, Solid State Electrochemistry and Electrical Characterization of Solids represent his main scientific activities.

\section{References}

1 A. Heller and B. Feldman, Chem. Rev. (Washington, DC, U.S.), 108 (2008) 2482

2 E. Katz, A.N. Shipway and I. Willner, Handbook of fuel cells : fundamentals, technology, and applications, Wiley, Chichester, England; New York, 2003

3 M. Tominaga, T. Shimazoe, M. Nagashima and I. Taniguchi, Electrochem. Commun., 7 (2005) 189

4 M. Tominaga, T. Shimazoe, M. Nagashima, H. Kusuda, A. Kubo, Y. Kuwahara and I. Taniguchi, J. Electroanal. Chem., 590 (2006) 37

5 W. Loeb, Biochem. Z., 17 (1909) 132
6 Y.B. Vasil'ev, O.A. Khazova and N.N. Nikolaeva, J. Electroanal. Chem. Interfacial Electrochem., 196 (1985) 127

7 A.E. Bolzan, T. Iwasita and W. Vielstich, J. Electrochem. Soc., 134 (1987) 3052

8 E.B. Makovos and C.C. Liu, Bioelectrochem. Bioenerg., 15 (1986) 157

9 R.R. Adzic, M.W. Hsiao and E.B. Yeager, J. Electroanal. Chem. Interfacial Electrochem., 260 (1989) 475

10 M.W. Hsiao, R.R. Adzic and E.G. Yeager, J. Electrochem. Soc., 143 (1996) 759

11 B. Lertanantawong, A.P. O'Mullane, W. Surareungchai, M. Somasundrum, L.D. Burke and A.M. Bond, Langmuir, 24 (2008) 2856

12 P. Schechner, E. Kroll, E. Bubis, S. Chervinsky and E. Zussman, J. Electrochem. Soc., 154 (2007) B942

13 W.M. Corbett and A.M. Liddle, J. Chem. Soc., (1961) 531.

14 L.D. Burke and P.F. Nugent, Gold Bull. (London), 30 (1997) 43

15 L.D. Burke and P.F. Nugent, Gold Bull. (London), 31 (1998) 39

16 Y.G. Zhou, S. Yang, Q.Y. Qian and X.H. Xia, Electrochem. Commun., 11 (2009) 216

17 C. Xiang, Q. Xie and S. Yao, Electroanalysis, 15 (2003) 987

18 B.K. Jena and C.R. Raj, Chem. Eur. J., 12 (2006) 2702

19 C. Jin and Z. Chen, Synth. Met., 157 (2007) 592

20 M. Comotti, C. Della Pina, R. Matarrese, M. Rossi and A. Siani, Appl. Catal., A, 291 (2005) 204

21 M. Comotti, C. Della Pina, E. Falletta and M. Rossi, Adv. Synth. Catal., 348 (2006) 313

22 M.J. Nicol, Gold Bull., 13 (1980) 46

23 L.H.E. Yei, B. Beden and C. Lamy, J. Electroanal. Chem. Interfacial Electrochem., 246 (1988) 349

24 S. Ernst, J. Heitbaum and C.H. Hamann, J. Electroanal. Chem. Interfacial Electrochem., 100 (1979) 173

25 S. Ernst, J. Heitbaum and C.H. Hamann, Ber. Bunsenges. Phys. Chem., 84 (1980) 50

26 F. Largeaud, K.B. Kokoh, B. Beden and C. Lamy, J. Electroanal. Chem., 397 (1995) 261

27 B. Beden, F. Largeaud, K.B. Kokoh and C. Lamy, Electrochim. Acta, 41 (1996) 701

28 A.J. Bard and L.R. Faulkner, Electrochemical methods: fundamentals and applications, John Wiley, New York, 2001

29 C.A. Toles, W.E. Marshall and M.M. Johns, Carbon, 37 (1999) 1207

30 Y.H. Li, S. Wang, Z. Luan, J. Ding, C. Xu and D. Wu, Carbon, 41 (2003) 1057 